\title{
COMPLEXITY REDUCTION OF CYCLOSTATIONARY SENSING TECHNIQUE USING IMPROVED HYBRID SENSING METHOD
}

\author{
Hikmat N. Abdullah ${ }^{a, *}$, Zinah O. Dawood ${ }^{b}$, Ammar E. Abdelkareem $^{a}$, \\ HADEEL S. ABED ${ }^{a}$ \\ a AL-Nahrain University, College of Information Engineering, Department of Information and Communication \\ Engineering, 10072 Jadiria, Baghdad, Iraq \\ ${ }^{b}$ University of Baghdad, Al-khwarizmi engineering college, Department of Information and Communication \\ Engineering, 10070 Jadriah, Baghdad, Iraq \\ * corresponding author: hikmat_04@yahoo.com
}

\begin{abstract}
In cognitive radio system, the spectrum sensing has a major challenge in needing a sensing method, which has a high detection capability with reduced complexity. In this paper, a low-cost hybrid spectrum sensing method with an optimized detection performance based on energy and cyclostationary detectors is proposed. The method is designed such that at high signal-to-noise ratio SNR values, energy detector is used alone to perform the detection. At low SNR values, cyclostationary detector with reduced complexity may be employed to support the accurate detection. The complexity reduction is done in two ways: through reducing the number of sensing samples used in the autocorrelation process in the time domain and through using the Sliding Discrete Fourier Transform (SDFT) instead of the Fast Fourier Transform (FFT). To evaluate the performance, two versions of the proposed hybrid method are implemented, one with the FFT and the other with the SDFT. The proposed method is simulated for cooperative and non-cooperative scenarios and investigated under a multipath fading channel. Obtained results are evaluated by comparing them with other methods including: cyclostationary feature detection (CFD), energy detector and traditional hybrid. The simulation results show that the proposed method with the FFT and the SDFT successfully reduced the complexity by $20 \%$ and $40 \%$ respectively, when 60 sensing samples are used with an acceptable degradation in the detection performance. For instance, when $E_{b} / N_{0}$ is $0 \mathrm{~dB}$, the probability of the detection of $P_{d}$ is decreased by $20 \%$ and $10 \%$ by the proposed method with the FFT and the SDFT respectively, as compared with the hybrid method existing in the literature.
\end{abstract}

KEYwords: Cognitive radio (CR), cyclostationary detector, Fast Fourier Transform (FFT), Sliding Discrete Fourier Transform (SDFT).

\section{INTRODUCTION}

Due to the expansion of remote gadgets and applications, the available electromagnetic radio range is becoming crowded. It has been recognized that due to the static allotment strategy of the spectrum, this allocated range will be under-utilized. "Spectrum holes" or "white Spaces" result from the unutilized part of the spectrum. Due to the limitation of the available spectrum and the inefficiency in its usage, a new communication paradigm is required to exploit the existing wireless spectrum opportunistically. Thus, cognitive radio (CR) has been recognized as the key enabling technology to overcome the spectrum under-utilization, in order to supply extremely reliable communication for all secondary users of the network 1]. A cognitive radio is a wireless technology that can automatically detect the available spectrum and changes its parameter accordingly. In this framework, cognitive users, called secondary users (SUs), can recognize the spectrum holes and use them to communicate among themselves without causing an interference to the license users, called primary users (PUs) [2].

Spectrum sensing is the main step of cognitive radio, this process is done by checking a spectrum band, and finding those channels not used by PU (licensed) users, which could be utilized by SUs 3 . Energy detection $\mathrm{ED}$, matched filter detection and cyclostationary detection techniques are basic types of spectrum sensing techniques. ED based sensing method is the most broadly used due to its simplicity and Low computational complexity. However, at a low SNR, ED has no capability to separate the PU signal from the noise. The matched filter maximizes the received SNR, so it could be viewed as an optimal detector. But, the matched filter has a demerit in that it needs an information about the PU signal characteristics, i.e., type of modulation, packet format, pulse shaping. If the CR doesn't have enough information about the PU signal, the performance of the matched filter is degraded. In such a situation, the cyclostationary detector can be used as a sub optimal detector. The cyclostationary detector can differentiate the PU signal from the noise since it exploits the periodicity 
property of a signal in its process. It can work in a low SNR condition 4 but it has a high computational complexity since it requires a long sensing time [5]. In order to improve the detection performance under fading and shadowing environments, cooperative spectrum sensing CSS is utilized. In CSS, many SUs sense the spectrum and each SU sends its local decision about the PU activity to the fusion centre (FC) during the transmission stage. In this stage, various types of coding algorithms can be used to guarantee the successful arrival of decisions. Finally, when these local decisions arrive to the FC, global decision about the status of the $\mathrm{PU}$ is taken [4, 6, 7].

Many works have addressed the complexity of the cyclostationary detection. In [5, 8, the complexity of the cyclostationary detection is reduced based on the selection of optimal parameters. In [9], the computational complexity of cyclostationary detection is addressed by reducing the test statistics sharing among multi antenna. In 10, the computational complexity is improved by a cyclic autocorrelation function CAF at only one cyclic frequency. In [11, a method is proposed for improving the computational complexity by splitting the autocorrelation into its real and imaginary parts and calculating two modified CAFs, before combining them in a final test statistic. Although the methods mentioned above reduce the computational complexity, they suffer from having a high degradation in the detection performance especially at low SNR values. Furthermore, none of them addressed the complexity during the autocorrelation process and during the conversion to the frequency domain except in [11], which addresses the autocorrelation process only.

In this paper, we proposed two methods to reduce the complexity of cyclostationary detectors based on FFT and SDFT with an acceptable detection performance. The proposed methods include a hybrid detection with complexity reduction in the autocorrelation process. The hybrid detection combines both the energy detector and the cyclostationary detector. The procedure of the proposed method is based on checking the received samples of the PU signal using the energy detection first. If it can detect the PU properly, there is no need to use a cyclostationary detector. But, if the ED has a false detection, then the cyclostationary detector is used to assist the detection. To save the complexity of the cyclostationary detector, only half samples of the PU signal are used for the autocorrelation process. To enhance the minor reduction in the detection performance due to neglecting the samples, the SDFT is used for the conversion into the frequency domain since it has a better accuracy than the FFT. Furthermore, the SDFT has a reduced complexity compared to the FFT, as will be discussed later.

The contributions of this paper are as follows: proposing a hybrid sensing method that can adapt its complexity according to the detection satisfactory level without the need for the SNR estimator circuitry. The complexity of the cyclostationary method is reduced by manipulating the number of sensing samples and using an efficient frequency domain conversion interchangeably. The equations of computational complexity of the proposed method are derived. The simulation results using MATLAB are obtained in AWGN and multipath fading channels in both cooperative and non-cooperative scenarios and the performance of the proposed methods are evaluated through comparing with the hybrid method in ref [4, traditional method of cyclostationary detector, and with the traditional method of an energy detector.

\section{EnERGY DETECTOR TECHNIQUE}

Due to its simplicity and low complexity, energy detection is considered as one of the broadly utilized spectrum sensing techniques. It doesn't necessitate to know the information about PUs signals structures. However, to correctly perform the detection, the information about the noise variance is needed. The ED has no ability to separate the PU signal from the noise at low SNR. The SU checks the range allocated to the PU and when it detects the absence of PU transmission, it starts the data transmission to its receiver. The received samples at the $\mathrm{CU}$ receiver are [4, 12]:

$$
x(n)=H \theta s(n)+n_{o}(n)
$$

where $x(n)$, is the received complex signal of the cognitive radio and it is a function of sample time, $s(n)$ is the transmitted signal of the primary user, $n_{o}$ is the Additive White Gaussian Noise (AWGN), and $H$ is the complex gain of the ideal channel, and $\theta$ is the activity indicator, which can take one out of two values as given in equation (2),

$$
\theta= \begin{cases}0 & \text { for } H_{0} \text { hypothesis } \\ 1 & \text { for } H_{1} \text { hypothesis }\end{cases}
$$

Hypothesis $H_{1}$ is referred to the $\mathrm{PU}$ in an active case, while hypothesis $H_{0}$ is referred to an inactive PU. By comparing the detector decision metric with a pre-set threshold $\lambda$, the false alarm and detection probabilities are evaluated. The decision metric $E_{j}$ is defined as the average accumulated energy for $j^{t h}$ SU of the captured samples during the monitoring window $W$.

$$
E_{j}=\frac{1}{N} \sum_{n=1}^{N s}|x(n)|^{2}
$$

where $N s$ is the number of samples $N s=W F s$, and $F s$ is the sampling frequency. The probability of a false alarm $P_{f}$ and probability of a detection $P_{d}$ are given by equations (4) and (5) respectively:

$$
\begin{aligned}
& P_{f}=\operatorname{pr}\left(E_{j}>\lambda \mid H_{0}\right) \\
& P_{d}=\operatorname{pr}\left(E_{j}>\lambda \mid H_{1}\right)
\end{aligned}
$$


Mathematically, the common equation for setting the threshold assuming $P_{f}$ is constant is given as in equation (6) [13].

$$
\lambda=\left(Q^{-1}\left(P_{f}\right)+\sqrt{N s}\right) 2 \sqrt{N s}(N s)^{2}
$$

where $Q^{-1}$ is the inverse of complimentary error function $Q($.$) .$

\section{Cyclostationary technique}

Cyclostationary characteristic identification is a method of discovering primary user transmissions by taking advantage of the cyclostationary features of the received signals 14. Earlier research endeavours exploit the cyclostationary merit of signal as a method for classification, which has been found to be better than match filtering and energy detection. Distinguished features are the number of signals, their modulation type, presence of interferer and symbol rate 14 .

The analysis of the sensing execution is done by the correlation process. The correlation can be enumerated by multiplying the received signal $x(n)$ and the identical delayed signal. To decide whether PU is present or absent, the sum of correlations is compared with a predetermined sensing threshold. PU is assumed to be present, if the sum of correlation is greater than the predetermined threshold, otherwise it is absent [4]. Because it can distinguish between the signal power and noise power, it performs better than energy detectors. However, it is very complex in that it requires a long time for processing, which generally breaks down the performance of Cognitive Radio. A signal is said to be cyclostationary if its autocorrelation is a periodic function with some period. This type of cyclostationary is called Second-order cyclostationary detection [14. A discrete cyclic autocorrelation function of a discrete time signal $x(n)$ with a fixed lag $l$ is defined as [15]:

$$
R_{x x}^{\alpha}(l)=\lim _{N s \rightarrow \infty} \frac{1}{N s} \sum_{m=0}^{N-1} x[m] x^{*}[m+l] e^{-j 2 \pi \alpha m \Delta m}
$$

where $N s$ is the number of samples of a signal $x[m]$ and $\Delta m$ is the sampling interval. By applying the discrete Fourier transform to $R_{x x}^{\alpha}(l)$, the cyclic spectrum (CS) is given as:

$$
S_{x x}^{\alpha}(f)=\sum_{l=-\infty}^{\infty} R_{x x}^{\alpha}(l) e^{-j 2 \pi f l \Delta l}
$$

The detection of the presence and absence of the signal is performed based on scanning the cyclic frequencies of its cyclic spectrum or its cyclic autocorrelation function. The decision is made very simple, i.e., at a given cyclic frequency, if the cyclic spectrum or its cyclic autocorrelation function $(\mathrm{CAF})$ is below the threshold level $\lambda$ as shown in equation (6), the signal is absent, otherwise the signal is present [14, 15].

\section{Hybrid SEnsing Method [4]}

To improve the detection probability of the CRU, a hybrid detector is suggested in [4]. It consists of energy and cyclostationary detectors. The energy detector is moderate compared to the cyclostationary detector. So, to verify whether the PU is present or not, the output of the PU transmitter first pushes through the ED. If the ED is not sure about the presence of PU, then a cyclostationary detector of first or second order is applied [4].

\section{The PROPOSED METHODS}

The proposed sensing methods use hybrid sensing, which consists of an energy detector and an improved cyclostationary detector. The main goal of the proposed methods is to reduce the computational complexity of the cyclostationary technique while maintaining a good probability of detection $P_{d}$. With this structure, since the energy detector has a satisfactory $P_{d}$ at high $E_{b} / N_{0}$ values, it is only used to save the complexity required by the cyclostationary detector. At low $E_{b} / N_{0}$ values, an improved version of the cyclostationary is used to reduce the computational complexity. Two methods are proposed to reduce the computational complexity of the cyclostationary spectrum sensing as shown in the following two sections:

\subsection{Hybrid PROPOSED METHOd USING FFT}

The procedure of the proposed method is: first, the QPSK signal, which is a PU signal, is processed through a one type of a channel, AWGN or Rayleigh multipath fading, then sensed using an energy detector. The accumulated energy of the PU signal $E_{j}$ is computed by the energy detector and passed to a comparator. If $E_{j}$ is greater than the threshold $\lambda$, the local sensing declares the PU presence without using the cyclostationary detector. Otherwise, the PU signal is sensed by using the cyclostationary detector. In this case, first, an autocorrelation function is applied to the samples of the PU signal, reducing the number of samples to half by selecting one sample and skipping the next alternatively. So, half the number of samples are entered to the autocorrelation process and this leads to a huge reduction in the computational complexity of the cyclostationary technique. Then, the result is converted to a frequency domain using traditional FFT to compute the cyclic spectrum CS and compare it with the pre-defined threshold $\lambda$. If it is greater than the threshold, the local sensing result is that the PU is present, otherwise the PU is absent. The procedure of the proposed method is shown as a flow chart in figure 1 .

\subsection{HYBRID PROPOSED METHOD USING SDFT}

This proposed method has the same procedure as is used in the first proposed method, but instead of using the FFT for the conversion to the frequency 


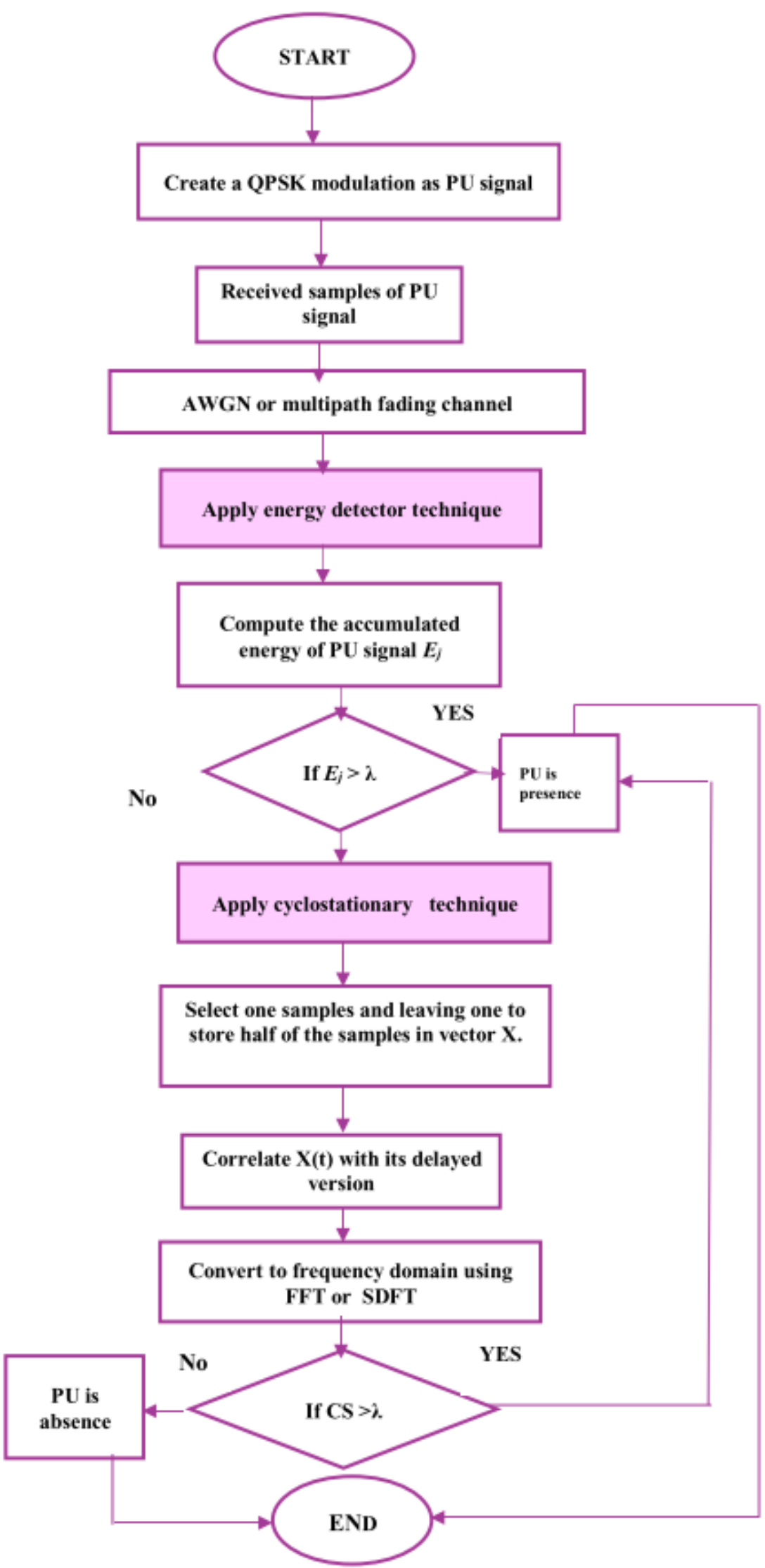

Figure 1. Procedure of the proposed methods. 
domain, sliding DFT (SDFT) is used. The SDFT can be greatly helpful to reduce the computational complexity [16]. The computational complexity of this method is $O(N s)$ for a single update, which is much lower than $O\left(N s \log _{2}(N s)\right)$ the FFT has [16]. Generally, given a discrete time signal $x$, at any time index $n$, the $k^{\text {th }}$ frequency bin of its M-point DFT is 17

$$
X_{n}^{k}=\sum_{m=0}^{M_{1}} W_{M}^{-k m} x_{n-M+1+m} \quad \forall k \in\{0,1, \ldots M-1\}
$$

Where $W_{M}=e^{j 2 \pi / M}$. Equation (9) can be transformed into its recursive equivalent

$$
X_{n}^{k}=W_{M}^{-k m}\left(X_{n-1}^{k}+x_{n}-x_{n-M}\right),
$$

The procedure of this proposed method is shown in Fig. 1. The probability of the detection of the proposed hybrid method $P_{d, \text { hybrid }}$ is given by [4]

$$
P_{d, \text { hybrid }}=1-\left(1-P_{d, E D}\right)\left(1-P_{d, c y c o}\right)
$$

where $P_{d, \text { hybrid }}$ is the probability of the detection of the proposed method, $P_{d, E D}$ is the probability of the detection in the energy detector stage, and $P_{d, c y c o}$ is the probability of the detection in the cyclostationary stage.

\section{The COMPUTATIONAL COMPLEXITy}

The computational complexity of cyclostationary technique of the traditional and proposed methods are computed as shown in the following equations:

$$
C x=C x_{\text {_auto }}+C x_{\text {_f }} \text { freq }
$$

where, $C x=$ total computational complexity of cyclostationary technique, $C x$ _auto $=$ computational complexity through the autocorrelation process, $C x \_$freq $=$ computational complexity when converting to frequency domain. In cyclostationary (traditional method) the computational complexity $C x 1$ is computed as follows:

According to [18], $C x_{\text {_auto }}$ is,

$C x \_$auto $=$No. of real multiplications + No. of real additions

$C x_{\text {auto }}=4 N s+4 N s-2=8 N s-2$

According [16], $C x \_$freq is,

$C x \_$freq $=$computational complexity in traditional

$\mathrm{FFT} C x \_$freq $=O\left(N s \log _{2}(N s)\right)$

So, 12 is transformed to

$$
C x 1=8 N s-2+O\left(N s \log _{2}(N s)\right)
$$

In the two hybrid proposed methods with the FFT and SDFT, the computational complexity $C x 2$ and $C x 3$, respectively, are computed as follows:

Since, half the number of samples $N s / 2$ is entered to the autocorrelation process, so, $C x_{\text {_auto }}$ in traditional method is transformed to: $C x_{\text {_auto }}=$
$2 N s+2 N s-2=4 N s-2$ in both $C x 2$ and $C x 3$. In the hybrid proposed method using the FFT, Cx_freq = $\mathrm{O}\left(\mathrm{Ns} \log _{2}(N s)\right)$, so $C x 2$ is:

$$
C x 2=4 N s-2+O\left(N s \log _{2}(N s)\right)
$$

In the hybrid proposed method using the SDFT, the $C x \_f r e q=O(N s)$, so $C x 3$ is:

$$
C x 3=2 N s+2 N s-2+O(N s)
$$

The computational complexity ratio of each method is computed as follows:

$C x 1 \_$ratio $=C x 1 / C \_\max , \quad C x 2 \_$ratio $=$ $C x 2 \bar{C} C \_$max,$C x 3 \_$ratio $=C x 3 / C \_$max , where, $C \_\max =$ maximum computational complexity of traditional method.

The computational complexity of cyclostationary in the hybrid method in $4 . C x 4$ is the same as the computational complexity in the traditional method $C x 1$, since the method does not take into consideration the complexity reduction in the cyclostationary stage. It should be noted that all equations of computational complexity focus only on computing the complexity in the cyclostationary stage of hybrid methods and not taking into consideration the computational complexity in the energy detector stage. Table 1 summarizes the equations of the computational complexity in traditional and proposed methods.

\section{SimUlation RESUlts AND DISCUSSION}

This section presents the simulation results of the performance of the proposed methods under cooperative and non-cooperative scenarios. The performance is tested under AWGN and Rayleigh multipath fading channels. We evaluate the performance of the proposed method by comparing it with a traditional hybrid method in reference [4, the cyclostationary feature detection (traditional method) and energy detector method. The procedure followed to produce the results below:

(1.) Creating QPSK modulation as a PU signal.

(2.) Adding a channel, which is an AWGN channel or ITU indoor channel model (A), like Rayleigh multipath fading

(3.) Applying one of the sensing methods shown in the previous sections.

(4.) There are two scenarios for the sensing process, in the non-cooperative scenario, single sensing method (single SU) is used to check the activity of the PU signal. In cooperative scenario, multiple SUs are used to sense.

(5.) Testing the sensing performance by computing the probability of the detection in equation (11) under various values of $E_{b} / N_{0}$ of PU signal. In the case of the cooperative scenario, the average probability of the detection over all SUs is computed. 


\begin{tabular}{ll}
\hline Method & Computational complexity equation \\
\hline Cyclostationary (traditional method) & $C x 1=4 N s+4 N s-2+O\left(N s \log _{2}(N s)\right)$ \\
Hybrid proposed method using FFT & $C x 2=2 N s+2 N s-2+O\left(N s \log _{2}(N s)\right)$ \\
Hybrid proposed method using SDFT & $C x 3=2 N s+2 N s-2+O(N s)$ \\
Hybrid method in [4] & $C x 4=4 N s+4 N s-2+O\left(N s \log _{2}(N s)\right)$ \\
\hline
\end{tabular}

TABle 1. Computational complexity.

(6.) Computing the computational complexity of each method from equations shown in Table 1 under various numbers of samples of the PU signal.

(7.) Plotting the obtained results.

The simulation parameters used are: QPSK modulation of the PU signal with a carrier frequency $F c=200 \mathrm{~Hz}$, sampling frequency $F s=4000 \mathrm{~Hz}$, and $P f=0.001$. The multipath fading used is "ITU indoor channel model (A)" [19. The simulation results are divided into two parts. The first part presents the results using a non-cooperative scenario, while the second is for the cooperative scenario.

\subsection{NON-COOPERATIVE SCENARIO}

Figure 2 shows the performance curves of a number of samples versus the computational complexity ratio in cyclostationary of hybrid proposed methods compared with the traditional method. It can be seen that the computational complexity increases as the number of samples increase in all methods, and the hybrid method using the FFT uses less computational power than the traditional method and hybrid method in 4], since only $50 \%$ of samples enter the autocorrelation process. It can also be noted that the computational complexity in the proposed hybrid method using the SDFT uses less computations than the FFT and traditional methods since the SDFT reduces the computational complexity and requires $O(N s)$ rather than $O\left(N s \log _{2} N s\right)$ required in FFT besides that $50 \%$ reduction of the sensing samples. It can be noted that the computational complexity in the hybrid method in [4] is the same as the computational complexity of traditional methods since it does not have any improvement in reducing the complexity during the cyclostationary process. For example, when the number of samples equals 60 , the computational complexity is reduced by $20 \%$, and $40 \%$ in proposed methods using FFT and SDFT respectively, compared with traditional methods.

Figures 3 and 4 show the proposed methods' performance curves in terms of the probability of detection $P_{d}$ versus $E_{b} / N_{0}$ in AWGN and Rayleigh multipath fading channels respectively, compared with hybrid method in 4], CFD (traditional method) and energy detector method. In both curves, $P_{d}$ increases as $E_{b} / N_{0}$ increases. In figure 3 the traditional method and hybrid method in 4 give the best results especially at low values of $E_{b} / N_{0}$. However, they have a high computational complexity, as shown in figure 2 , because they require a long time for processing. The hybrid method in [4 has a high complexity at low values of $E_{b} / N_{0}$, since it firstly utilizes the energy detector for sensing and then uses the cyclostationary detector with the full number of samples. But, the proposed methods reduce the computational complexity and give a good detection performance. The price we pay for the complexity reduction is a slight loss in $P_{d}$ as compared with the traditional method, also, it can be seen that although the proposed method using the SDFT hasa greater reduction in the computational complexity than the other proposed methods that use the FFT, it also gives a good detection performance, especially at low values of $E_{b} / N_{0}$, since the SDFT has a higher accuracy than the FFT and the performance of other methods becomes the same as the method that uses the SDFT at $E_{b} / N_{0}$ equals $6 \mathrm{~dB}$. As shown in the figure, when $E_{b} / N_{0}$ equals $0 \mathrm{~dB}, P_{d}$ is decreased by $20 \%$ and $10 \%$ using proposed methods that use the FFT and SDFT respectively, as compared with the hybrid method in [4] and increased by $30 \%$ and $40 \%$ respectively, as compared with the energy detector. At higher values of $E_{b} / N_{0}, P_{d}$ of the energy detector becomes almost the same as for other methods. In figure 4 the performance curves are the same as in figure 3 but with a degradation in the detection performance because of multipath fading. The energy detector has worse results at low values of $E_{b} / N_{0}$ and the proposed methods also give acceptable values of $P_{d}$ especially in the proposed method that uses the SDFT. For example, at $E_{b} / N_{0}$ equals $3 \mathrm{~dB}, P_{d}$ decreases by $28 \%$ and $5 \%$ in the proposed methods that use the FFT and SDFT respectively, as compared to traditional and hybrid methods in [4]. Also, $P_{d}$ of the proposed methods increases by $50 \%$ and $75 \%$ respectively as compared to the energy detector at the same value of $E_{b} / N_{0}$. The difference between methods when $P_{d}$ equals 1 can be compared in the case of the fading channel as shown in figure 4 Since the fading channel is more realistic than the AWGN, it can be seen that when $P_{d}$ becomes $1, E_{b} / N_{0}$ required by the energy detector (traditional method), cyclostationary (traditional method), hybrid method in refrence [4, proposed method using FFT, and proposed method using SDFT are $9.1 \mathrm{~dB}, 5.1 \mathrm{~dB}, 6 \mathrm{~dB}$, $6 \mathrm{~dB}$, and $6 \mathrm{~dB}$ respectively. We can further improve the detection performance in all methods under fading channel by using the cooperative scenario as shown in the following section. 


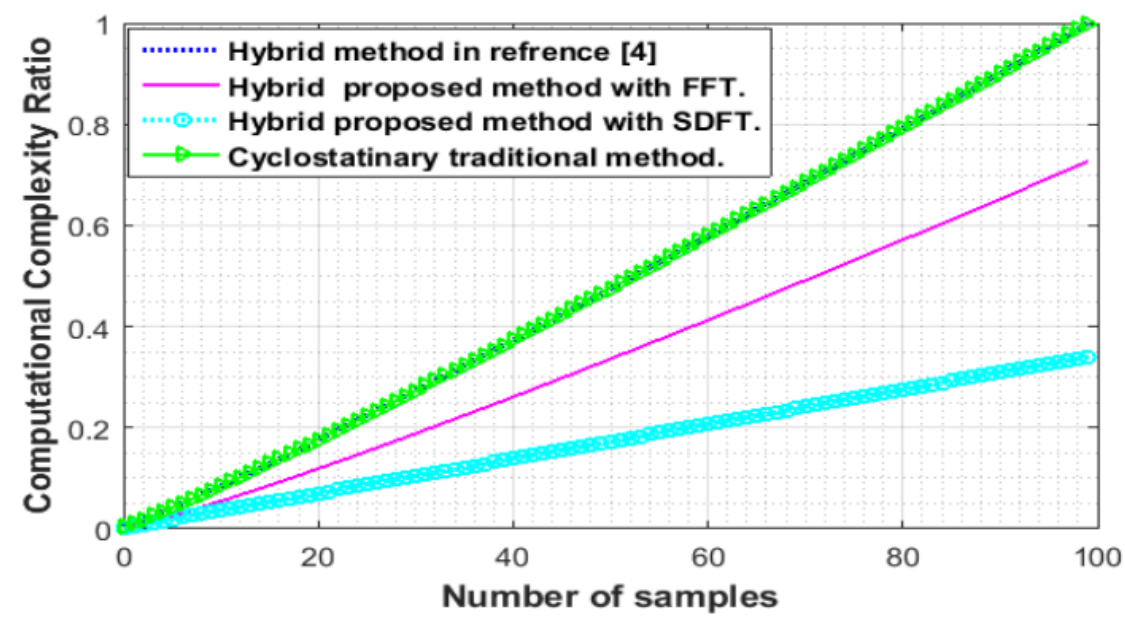

FiguRE 2. The performance curves of number of samples versus computational complexity ratio.

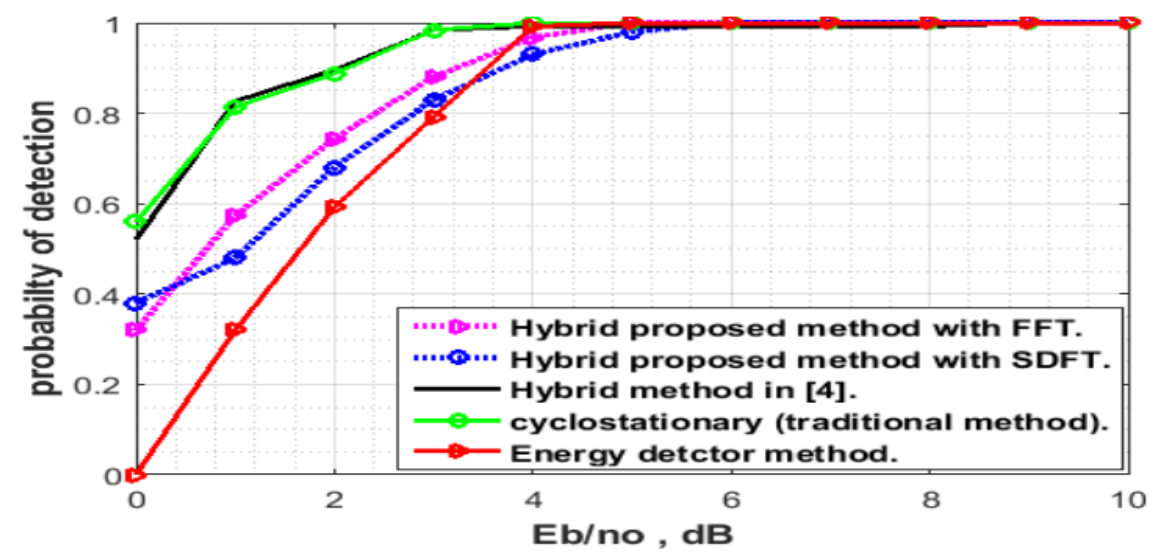

FIGURE 3. The performance curves of traditional and hybrid methods in AWGN channel.

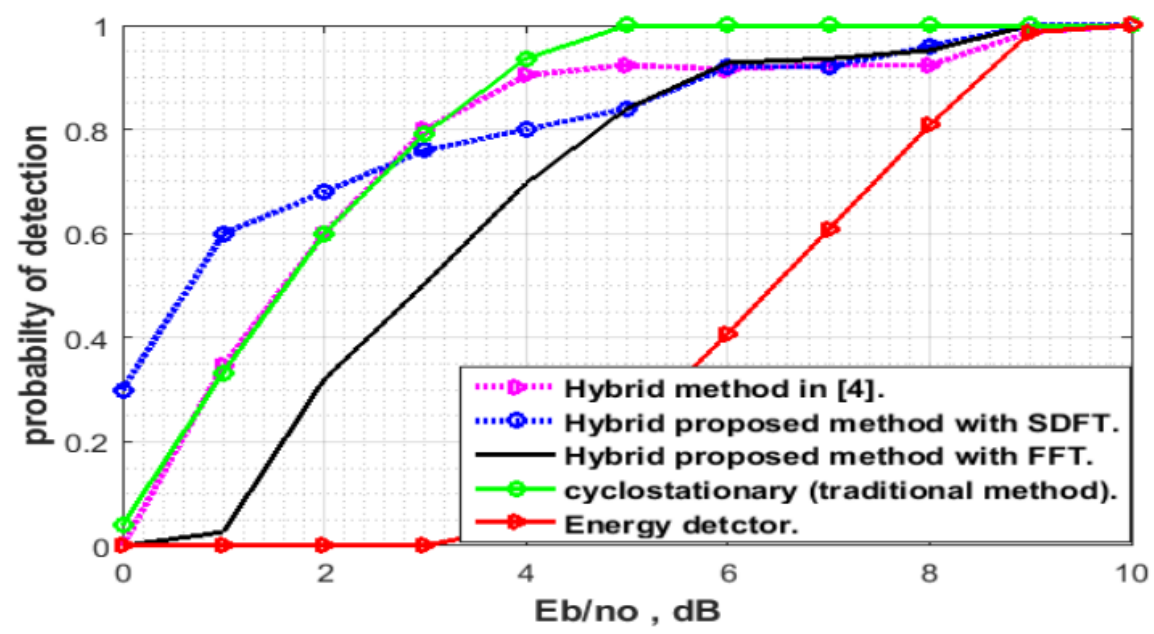

FiguRE 4. The performance curves of traditional and hybrid methods in Rayleigh multipath fading channel. 


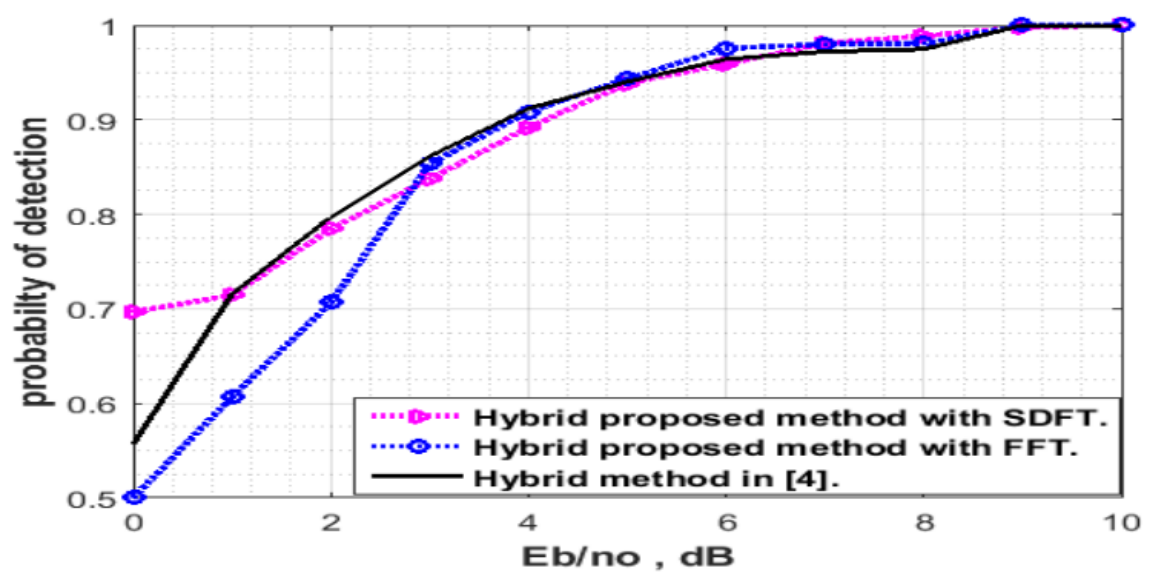

Figure 5. The performance curves of the proposed methods of $P_{d}$ versus $E_{b} / N_{0}$ in cooperative scenario compared with hybrid method in [4].

\subsection{Cooperative SCEnARIO}

In the cooperative scenario, the effect of fading is reduced. In this scenario, we assumed that $3 \mathrm{CUs}$ are used to sense the spectrum and one of them is suffering from multipath fading. Figure 5 shows the performance curves of the proposed methods in terms of $P_{d}$ versus $E_{b} / N_{0}$ as compared with the hybrid method in 4 . In figure 5 , the detection performance of the proposed method using the FFT is approximately the same as for the hybrid method in [4]. For example, at $E_{b} / N_{0}$ equals $0 \mathrm{~dB}, P_{d}$ is reduced by $5 \%$ in the proposed method using the FFT as compared to the hybrid method 4. It can be seen that the proposed method using the SDFT outperforms other methods, for example, when $E_{b} / N_{0}$ equals $0 \mathrm{~dB}, P_{d}$ in the proposed method using the SDFT is increased by $15 \%$, and $20 \%$ compared to the hybrid method in 4 and proposed method using the FFT respectively. We conclude that the proposed method operating in the cooperative scenario is better than that in the non-cooperative one and it is very efficient, since it significantly reduces the computational complexity.

\section{Conclusions}

In this paper, the performance of CR in terms of detection performance in cooperative and non-cooperative scenarios under AWGN and multipath fading channels is investigated. The performance is evaluated using traditional and hybrid methods available in literature, energy detection, and improved hybrid (proposed methods) using the FFT and SDFT. The equations of computational complexity are derived. It was observed that the traditional and hybrid methods in the literature have approximately the same performance and they have the best performance, but they have a high computational complexity. The energy detector has the worst detection performance. However, the proposed methods reduce the computational complexity by using only a half number of samples in the autocorrelation process and using the SDFT in the second proposed method with a little loss in the detection performance. The proposed methods become more efficient when the cooperative scenario is assumed. This technique can be developed in the future by taking other types of fading channels and using double threshold in the sensing process to achieve an improvement in the probability of detection. Also, the transmission stage and choosing an appropriate coding techniques can be taken into consideration.

\section{REFERENCES}

[1] A. S. Khobragade, R. D. Raut. Hybrid spectrum sensing method for cognitive radio. International Journal of Electrical and Computer Engineering 7(5):2683 2695, 2017. DOI:10.11591/ijece.v7i5.pp2683-2695

[2] A. Sepasi Zahmati, X. Fernando, A. Grami. A hybrid spectrum sensing method for cognitive sensor networks. Wireless Personal Communications: An International Journal 74:953 - 968, 2014. DOI:10.1007/s11277-013-1332-4.

[3] M. Kustra, K. Kosmowski, M. Suchański. Performance of hybrid sensing method in environment with noise uncertainty. Journal of Telecommunications and Information Technology 1:51 - 57, 2018. DOI:10.26636/jtit.2018.123117

[4] K. Yadav, S. Dhar Roy, S. Kundu. Hybrid cooperative spectrum sensing with cyclostationary detection for cognitive radio networks. In 2016 IEEE Annual India Conference (INDICON), pp. 1 - 6. 2016. DOI:10.1109/INDICON.2016.7839118.

[5] H. Arezumand, P. Azmi, H. Sadeghi. A low-complexity cyclostationary-based detection method for cooperative spectrum sensing in cognitive radio networks. International Journal of Information 85 Communication Technology Research 3(3):1 - 10, 2011. DOI:10.1109/iccke.2011.6413370

[6] K. Vasudevan, A. P. K. Reddy, G. K. Pathak, S. Singh. On the probability of erasure for mimo-ofdm. Semiconductor Science and Information Devices 2(1), 2020. DOI:10.30564/ssid.v2i1.1689. 
[7] K. Vasudevan. Coherent detection of turbo coded OFDM signals transmitted through frequency selective Rayleigh fading channels. In 2013 IEEE International Conference on Signal Processing, Computing and Control, ISPCC 2013, pp. 1 - 6. 2013. DOI:10.1109/ISPCC.2013.6663392

[8] D. Shen, D. He, et al. An improved cyclostationary feature detection based on the selection of optimal parameter in cognitive radios. Journal of Shanghai Jiaotong University (Science) 17(1):1 - 7, 2012. DOI:10.1007/s12204-012-1222-z

[9] D. Cho, S. Narieda, K. Umebayashi. Low computational complexity spectrum sensing based on cyclostationarity for multiple receive antennas. IEICE Communications Express 7(2):54 - 59, 2018. DOI:10.1587/comex.2017XBL0167.

[10] S. Narieda. Computational complexity reduction for signal cyclostationarity detection based spectrum sensing. In Proc. IEEE Int'l Symp. on Circuits and Systems (IEEE ISCAS 2017), pp. 1 - 4. 2017. DOI:10.1109/ISCAS.2017.8050564

[11] D. Allan, L. Crockett, R. Stewart. A low complexity cyclostationary detector for OFDM signals. In 2017 New Generation of CAS (NGCAS), pp. 253-256. 2017. DOI:10.1109/NGCAS.2017.19

[12] M. Emara, H. Ali, S. Khamis, F. Abd El-Samie. Spectrum sensing optimization and performance enhancement of cognitive radio networks. Wireless Personal Communications 86, 2015. DOI:10.1007/s11277-015-2962-5.
[13] S. Atapattu, C. Tellambura, H. Jiang. Energy Detection for Spectrum Sensing in Cognitive Radio. Springer-Verlag, New York, 2014. DOI:10.1007/978-1-4939-0494-5.

[14] W. Adigwe, O. R. Okonkwo. A review of cyclostationary feature detection based spectrum sensing technique in cognitive radio networks. E3 Journal of Scientific Research 4(3):41 - 47, 2016. DOI:10.1007/s11277-015-2962-5

[15] K. Po, J. Takada. Signal detection based on cyclic spectrum estimation for cognitive radio in IEEE 802.22 WRAN system. Technical report of ieice, The institute of electronics, information and communication engineers, 2007.

[16] P. Kruczek, J. Obuchowski. Cyclic modulation spectrum - An online algorithm. In IEEE 24th Mediterranean Conference on Control and Automation (MED), pp. 361 - 365. 2016. DOI:10.1109/MED.2016.7535994

[17] B. Han, H. Schotten. A cost efficient and flexible cyclostationary feature detector based on sliding discrete fourier transform for cognitive spectrum sensing. In IEEE 24th International Conference on Telecommunications (ICT). 2017. DOI:10.1109/ICT.2017.7998263

[18] S. Narieda. Low complexity cyclic autocorrelation function computation for spectrum sensing. IEICE Communications Express 6(6):387 - 392, 2017. DOI:10.1587/comex.2016XBL0211

[19] M. Pätzold. Mobile Fading Channels. John Wiley \& Sons, Ltd, 2000. DOI:10.1002/0470847808. 